Mots. Les langages du politique

\title{
L'unité aymara par-delà les frontières ?
}

Ethnicité et sécurité dans les Andes (Chili, Bolivie, Pérou)

The Aymara unity beyond the borders? Ethnicity and security in the Andes

(Chile, Bolivia, Peru)

La unidad aymara allá de las fronteras? Etnicidad y la seguridad en los Andes

(Chile, Bolivia, Perú)

\section{Lætitia Rouvière}

\section{OpenEdition}

Journals

Édition électronique

URL : https://journals.openedition.org/mots/22128

DOI : $10.4000 /$ mots. 22128

ISSN : 1960-6001

Éditeur

ENS Éditions

Édition imprimée

Date de publication : 30 novembre 2015

Pagination : 83-98

ISBN : 978-2-84788-776-1

ISSN : 0243-6450

\section{Référence électronique}

Lætitia Rouvière, «L'unité aymara par-delà les frontières ? », Mots. Les langages du politique [En ligne], 109 | 2015, mis en ligne le 30 novembre 2017, consulté le 23 avril 2022. URL : http:// journals.openedition.org/mots/22128; DOI : https://doi.org/10.4000/mots.22128 


\section{L'unité aymara par-delà les frontières? Ethnicité et sécurité dans les Andes (Chili, Bolivie, Pérou)}

«Indien est le nom avec lequel ils nous ont soumis, Indien sera le nom avec lequel nous nous libérerons !»Cette phrase célèbre, attribuée à un rebelle péruvien en 1922 et utilisée par certains mouvements kataristes boliviens (Albó, 2000, p.50), est emblématique des usages renouvelés de l'ethnicité en Amérique latine : dans une logique plus générale d'inversion du stigmate (Goffman, 1975), elle consiste à affirmer positivement une identité historiquement construite dans un sens péjoratif par les groupes dominants (Bourdieu, 1980). En Amérique du Sud et dans le cas aymara notamment, la Bolivie fait souvent figure de modèle ; la rénovation politique de l'indianité y a pourtant été portée par des dynamiques nationales propres, telles que la force historique des revendications autochtones et les changements politiques initiés depuis l'accession au pouvoir du président d'origine aymara Evo Morales (Do Alto, Stefanoni, 2008; Alvizuri, 2012). Il faut donc se défaire de l’idée d'homogénéité de la représentation des populations indigènes sur le continent (Andolina et al., 2009, p.14) et de cohésion interne de la néo-indianité (Galinier, Molinié, 2006), en appréhendant les usages discursifs de l'indianité dans la diversité des situations nationales, des supports institutionnels ou associatifs et des réseaux sociaux dans lesquels ils se déploient.

Ainsi, la constitution d'un discours transfrontalier sur l'identité aymara doit être comprise comme un processus répondant à diverses interactions et non pas simplement comme une présentation du groupe qui correspondrait à son (ses) identité(s) sociales (Charaudeau, 2009). Nous analysons ici les ressorts pratiques de la mise en indianité des discours locaux dans les Andes à partir d'un cas : l'Alliance stratégique Aymaras sans frontières (ASASF ou Alliance), formée par 57 municipalités rurales depuis 2001 entre le Chili, le Pérou et la Bolivie (voir carte). Les frontières entre ces trois États sont le produit de la guerre du Pacifique (1879-1884), ayant conduit à l'annexion par le Chili des provinces d'Arica et Tarapacá, auparavant péruviennes, et d'Antofagasta, qui fournissait 
à la Bolivie son unique accès à l'océan Pacifique. Il s'agit d'un espace diplomatiquement conflictuel, les délimitations territoriales étant aujourd'hui l'objet de deux procès au Tribunal international de la Haye (Rouvière, Perrier-Bruslé, 2015). La logique transnationale de légitimation de cette action locale est donc portée par un discours novateur, parce que commun de tous les côtés de cette triple frontière, sur ce que signifie être Aymara. Malgré un processus national de reconnaissance des droits des populations indigènes tardif en comparaison avec le Pérou et plus encore la Bolivie (Rouvière, 2012), c'est paradoxalement la partie chilienne de cette Alliance qui détient le leadership de l'action transfrontalière, la partie bolivienne - de par le niveau économique du pays assurant la réception des fonds internationaux et la partie péruvienne étant plus réticente à une alliance de long terme avec les Chiliens. Nous concentrons donc ici le propos sur les principaux acteurs de la légitimation de l'Alliance et producteurs de son discours officiel : les maires ruraux chiliens et les agents des institutions internationales.

L'accès à des réseaux transnationaux, notamment les institutions financières internationales, confère aux maires ruraux une visibilité et des ressources politiques inédites (Morin, Santana, 2003 ; Bellier, 2012), en même temps qu'il les amène à relayer les discours portés par leurs financeurs (Lavaud, Lestage, 2006). Pour comprendre l'émergence de ce discours, nous nous appuyons principalement sur l'analyse d'entretiens et de rapports publics. Les entretiens cités ont été conduits avec des autorités locales et un agent de la Banque interaméricaine de développement (BID) en 2006 et 2007, au moment de l'approbation du projet de l'Alliance par la BID. Certains rapports des bailleurs de fonds permettent de comprendre le contexte de naissance des discours transnationaux sur l'ethnodéveloppement dans les années quatre-vingt-dix; ceux concernant directement le cas étudié proviennent d'archives de l'ASASF et de la BID entre 2001 et 2011. La mise en cohérence des discours locaux avec les attentes internationales concernant les populations autochtones peut être observée au gré des interactions entre acteurs, cette dynamique venant confirmer le rôle premier de «l'environnement» dans la constitution du discours (Maingueneau, 2010).

Ces syncrétismes discursifs se produisent en particulier par l'appropriation, de la part des maires aymaras, du paradigme de développement avec identité, ou ethnodéveloppement. Tel qu’il est promu et construit par les agences internationales, ce dernier comporte une forte dimension normative, la culture y étant notamment utilisée comme un produit et comme une institution (Radcliffe, Laurie, 2006). Toutefois, les normes portées par les projets qui s'inscrivent dans ce cadre ne peuvent être comprises qu'en tenant compte de leur réinterprétation locale. Le discours émergent sur une identité aymara transfrontalière repose en l'occurrence sur l'idée d'unité culturelle autour de la frontière et de capacité de dialogue et de consensus inhérente aux communautés 


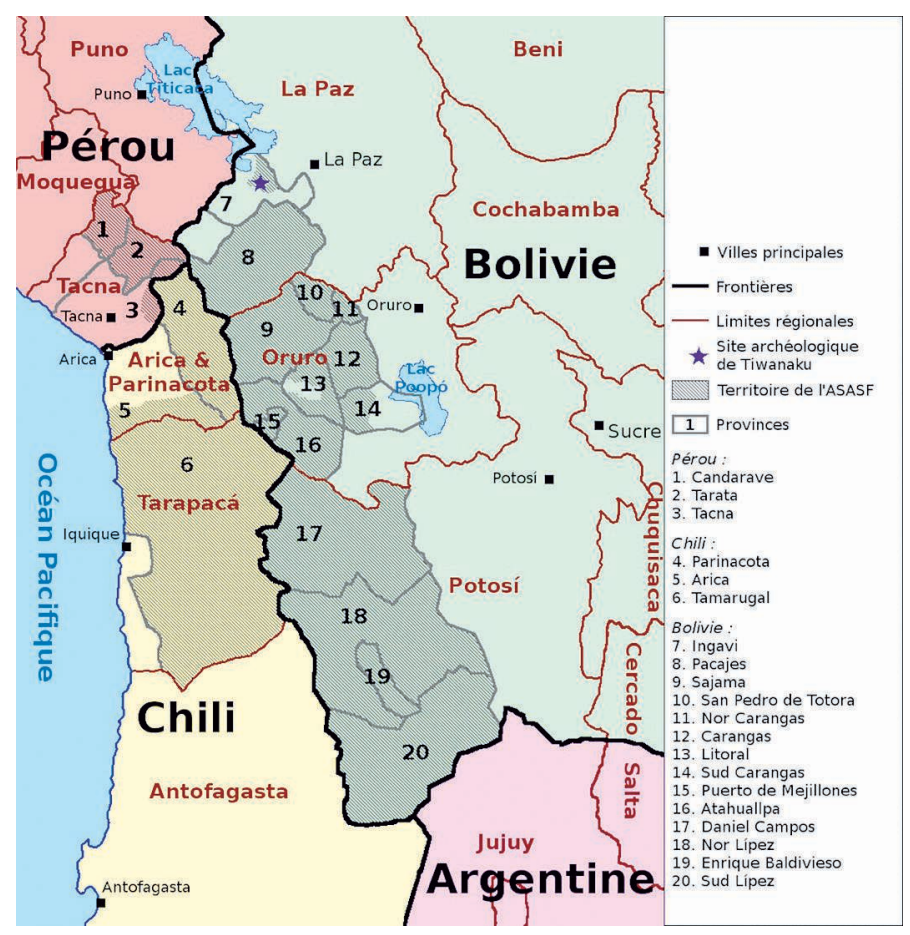

Le territoire de l'ASASF

Carte : Lætitia Rouvière $(C)$

andines, en écho aux présupposés diffusés par les agences internationales (1). Un projet local de développement économique «trinational» devient ainsi celui du «Monde Aymara » (2). Plus encore que satisfaire les a priori externes sur les dynamiques culturelles autochtones, ce discours est complété par et rend possible un second niveau de discours concernant les dimensions sécuritaires du développement sur un altiplano andin. L'ethnicisation du transfrontaliervient alors agir dans les zones frontalières aymaras pour mieux les sécuriser (3).

\section{Nous ne sommes qu'Un. Communauté andine et consensus transfrontalier}

La présentation par les élites locales du groupe transfrontalier aymara en tant qu'unité homogène n'a rien d'évident. Afin de saisir l'évolution des discours portés par et sur l'action transfrontalière aymara, il faut commencer par comprendre comment se présuppose, puis se construit le consensus dans les 
localités autochtones. La constitution locale d'un discours ethnique autour de la frontière se réfère constamment à ces présupposés : c'est dans un mouvement dialogique (Bres, Nowakowska, 2005) que s'établit progressivement la mise en scène discursive de l'unité transfrontalière.

L'intervention directe des bailleurs de fonds internationaux dans les communes rurales andines à partir du milieu des années quatre-vingt-dix renvoie à une période de prise de conscience des effets pervers des politiques d'ajustement structurel dans les milieux les plus défavorisés (Stiglitz, 1998) ${ }^{1}$. Les stratégies qui doivent compenser ces effets s'appuient sur la formation des populations et de leurs représentants locaux à l'élaboration de plans de développement (Hibou, 1998; Andolina et al., 2009, p. 80-81), alors que commencent également à se diffuser des réflexions sur l'insertion des dimensions culturelles dans la définition du développement. Cette redéfinition s'accompagne d'une augmentation du recours aux espaces locaux comme lieux d'action privilégiés pour la lutte contre la pauvreté, notamment dans l'intention d'adapter les interventions des agences aux demandes des populations bénéficiaires (Banco Mundial, 1991). Dans le cas des populations indigènes, ces idées prennent la forme du développement avec identité, incluant la capitalisation du potentiel naturel, culturel et social, qui présente un certain nombre d'avantages comparatifs (Deruyttere, 2006).

Selon les documents publiés par les institutions internationales, le capital social indigène constituerait une base efficace pour l'accroissement du capital économique. Un document de la Banque mondiale sur ce sujet indique ainsi dès 1996 :

Nous savons que le capital social, les valeurs partagées, les règles d'un comportement social correct et un sens commun de la responsabilité civique et de l'identité, sont les plus fortes caractéristiques culturelles des communautés indigènes. La Banque cherche à construire sur la base de cette force. (Partridge et al., 1996, p. 32)

En affirmant l'évidence d'un consensus qui serait issu de relations de confiance et de pratiques politiques traditionnelles dans les communautés andines, ce présupposé tend à lisser la réalité sociale des groupes en question. Les principes de réciprocité et d'accès aux ressources collectives font certes partie de l'organisation sociopolitique andine, mais sans pour autant exclure les phénomènes internes d'exclusion, de conflits et de domination (Bouysse-Cassagne, 1987). À ce propos, S. Radcliffe et N. Laurie remarquent que les institutions communautaires représentent « une formalité non-étatique qui jouit d'une légitimité considérable dans la pensée des agences multilatérales pour promouvoir la société civile et la participation sous la forme de politiques de “bonne gouvernance” néolibérale ( Radcliffe, Laurie, 2006, p. 243).

1. Voir notamment Banco Mundial, 1987. 
Bien que les communautés aient totalement disparu dans le nord du Chili, cette perception s'étend dans notre cas d'étude jusqu'à une confusion entre institutions communautaires et municipales.

En effet, le cas de l'ASASF illustre bien cette dynamique de mise en lien direct entre acteurs locaux et bailleurs de fonds internationaux. Après un accord passé entre maires, les premiers pas de l'Alliance se réalisent en relation avec des agents de la Banque mondiale, par l'organisation de plusieurs rencontres et séminaires entre 2001 et 2003 , au cours desquels circule notamment un document intitulé «Une proposition d'empowerment pour les Aymaras» (Banco Mundial, 2002). La BID prend ensuite le relais avec l'approbation, en 2007, du projet « récupération, développement et mise en valeur du patrimoine naturel et culturel aymara », qui s'appuie sur le modèle du développement avec identité pour promouvoir la coopération transfrontalière. Le soutien de la BID au projet de l'ASASF se fonde alors sur l'existence supposée d'un capital social aymara entendu comme une forme d'organisation sociale, politique et économique participative et délibérative, mais dont le potentiel ne serait pas accompagné de stratégies correspondantes (BID, 2007). Un entretien avec le responsable du projet pour la BID à Washington nous enseigne que l'existence d'un consensus entre les dirigeants de l'ASASF n'est pas questionnée, une tradition de concertation œuvrant, selon notre interlocuteur, en faveur de la prise de décision collective :

Il y a des possibilités de mener à bien le projet, parce qu'ils ont une tradition, [...] ils savent se concerter, travailler ensemble, ils ont trouvé un consensus sur la proposition, etc., et l'impression que nous avons toujours eue ici c'est que cette action collective entre les représentants des municipalités des trois pays était très forte. ${ }^{2}$

Les Aymaras jouiraient donc d'une propension particulière à la coopération, attribuée à une origine commune, des liens transfrontaliers préexistants et une organisation sociopolitique traditionnelle. Ainsi retrouve-t-on dans les discours portés sur l'Alliance une omission de relations internes fortement concurrentielles et hiérarchiques : l'Alliance est effectivement le projet d'un homme, le maire de la commune chilienne de Putre et président de l'ASASF, qui nous affirmait en entretien que «[t]ant que Francisco Humire est là, cette alliance va survivre. Quand ils voudront sortir Francisco Humire, cette alliance mourra. Et ça, je vous le dis directement »³.

Les dirigeants locaux saisissent aisément la niche que présente la mise en scène d'une identité transfrontalière essentialisée, dans une logique assumée d'adaptation des sociétés aymaras aux exigences des bailleurs de fonds. Un document produit par l'Alliance en 2003 réinterprète les fondements du regroupement des maires en 2001 par « [l]'existence d’un passé historique,

2. Entretien avec le responsable du projet pour la BID à Washington, 2007.

3. Entretien avec le maire de la commune chilienne de Putre et président de l'ASASF, 2006. 
d'une langue, d'une cosmovision, d'un espace territorial et d'une origine communs ». Dès lors, l'objectif affiché de l'Alliance est d'opter pour le développement avec identité afin de faire face aux effets de la mondialisation. Le même document affirme :

Le modèle de développement aymara durable aura pour stratégie l'articulation des gouvernements locaux des trois pays et sera un instrument propre qui permettra une plus grande modernisation et dynamisation des territoires. Cependant, atteindre cet objectif est seulement possible si l'acteur principal du modèle s'adapte aux changements associés à la mondialisation. Dans ce sens, cela requiert que l'homme et la femme aymara s'adaptent à la culture dominante de la globalisation et qu'ils développent la capacité d'initiative pour la création de micro-entreprises. (ASASF, 2003)

Le développement est donc défini par les membres de l’ASASF comme le résultat d'une adaptation des habitants de la zone aux exigences de la «culture dominante de la globalisation », caractérisée par l'esprit d'initiative et l'insertion sur le marché, mais impliquant prioritairement une identification croissante à l'ethnie aymara. En 2006, la reconstruction discursive des origines du projet est confirmée par le président de l'Alliance :

Donc j'ai pensé qu'ici il fallait affronter les problèmes tous ensemble. Et que nous avions un avantage. Qui était que les communes frontalières des trois pays appartenaient à la culture millénaire aymara. C'était ça notre base pour agir. C'était ça notre potentialité, et qu'il fallait s'accrocher à ça. ${ }^{4}$

L’idée d’une culture millénaire aymara évoquée par le dirigeant chilien mobilise dorénavant une mémoire ancrée dans un territoire devenu transfrontalier. L'existence de la ligne de démarcation permet également au groupe de se positionner contre les nationalismes, un référent d'opposition nécessaire à la construction de toute identité (Héritier, 1977; Charaudeau, 2009). Le leader de l'ASASF affirme ainsi dans le même entretien que «[c]ela n'existe pas dans le monde aymara. Ça n'existe pas. Ces problèmes se rencontrent à La Paz, à Oruro, à Cochabamba, à Santa Cruz, à Lima, à Santiago. Ici dans la région de frontière, nous ne sommes qu'Un. Tout ce qui est de trop, ce sont les frontières ».

\section{Les figures de l'Un. De l'Alliance trinationale au Monde Aymara}

La présentation ethnique du groupe se généralise et s'approfondit au cours des années d'existence de l'Alliance. Pourtant, au cœur de la construction de cette image du monde aymara existent des nuances dans les formes de présentation en fonction des interlocuteurs. Cette dynamique classique de construction

4. Entretien avec le maire de Putre, 2006. 
de l'ethnicité (Barth, 1995; Balandier, 1980) confère une marge de manœuvre aux maires andins par le jeu des interactions sociales à des échelles multiples. L'appropriation locale des discours dominants se réalise ainsi de manière progressive, stratégique et pragmatique. Au gré des interactions avec des agences internationales de financement, l'aspect culturel est valorisé de manière croissante : le lieu, le nom, le peuple, le territoire, la cosmovision, puis le monde aymara font progressivement leur apparition dans la présentation du groupe andin par les maires.

La réinterprétation de l'événement qui a vu naître l'ASASF est une première illustration de l'évolution des discours portés sur la coopération transfrontalière sur l'altiplano. À l'occasion de la seconde feria régionale andine (FERAN) de novembre 1999 à Putre (Chili), le maire lance pour la première fois l'idée d'une mise en commun du potentiel touristique, productif et commercial de la région frontalière. Le contexte se prête bien à cette proposition : la figure des ferias transfrontalières est l'une des pratiques les plus visibles du quotidien de l'altiplano; la FERAN, tenue annuellement, présente toutefois un profil original, comme nous le rapporte un ancien fonctionnaire de la municipalité de Putre en entretien :

La FERAN est une feria régionale andine qui se réalise à Putre, qui trouve sa genèse dans la période du gouvernement militaire. La FERAN, au début, convoquait des entreprises privées pour qu'elles puissent offrir leurs produits et montrer au monde un lieu différent, comme Putre. ${ }^{5}$

Les objectifs de l'alliance répondent à l'orientation originelle de cette feria, c'est-à-dire à une concentration sur les échanges commerciaux plutôt que sur des considérations d'ordre culturel ${ }^{6}$. Le projet initial de l'Alliance, nommé en 2000 "Circuits thématiques intégrés, Route altiplanique » ${ }^{7}$, est principalement de nature touristique. En tant que tel, le projet n'est toutefois pas éligible à un soutien de la part des organismes internationaux :

Eux au début, ils étaient très, très concentrés sur le secteur privé, le commerce... Non, l'idée est de donner une impulsion supplémentaire à ce qu'il y a, à des expériences réussies dans le secteur public-privé, et là où il n'y en n'a pas, voir comment ça peut se développer [...]. L'idée est d'identifier quelques initiatives qui ensuite puissent être présentées au financement dans une étape postérieure, à la BID ou à d'autres donateurs, etc... ${ }^{8}$

5. Entretien avec un fonctionnaire de la municipalité de Putre, 2006.

6. Asociación de municipalidades rurales andinas de tacna, Centro de promoción y asistencia para el desarrollo, 2002, «Programa trinacional de desarrollo fronterizo. Objetivos y programa de intermediación », La Paz, 16 janvier.

7. ASASF, 2007; entretien avec le consultant chilien en charge de l'élaboration du projet BID en 2005, 2006.

8. Entretien avec le responsable du projet pour la BID. 
L'aspect touristique, s’il est maintenu dans les faits, devra s'insérer dans des objectifs plus larges de mise en valeur du patrimoine aymara. Créée à l'origine pour favoriser le commerce sur l'altiplano chilien, la FERAN est alors réinventée comme un lieu d'intégration subrégionale et de réunion culturelle des populations indigènes des trois pays. L'événement portant l'Alliance voit également sa dénomination évoluer : la Feria régionale de l'Altiplano devient la Feria régionale andine et en 2006, par la même occasion, une Feria de la chanson andine. Les usages locaux et indigènes des frontières étatiques se mettent donc en place par la récupération de figures relativement anciennes de pratiques transfrontalières telles que les ferias, mais cette fois organisées sous la forme d'expositions culturelles à destination des marchés internationaux. La frontière devient ainsi un véritable support d'invention de la tradition (Hobsbawm, 1995). La notion de patrimoine devient progressivement centrale et s'appuie sur une valorisation de la culture aymara, celle-ci devant au préalable être nommée, située et caractérisée par un peuplement et une religiosité spécifiques.

L'évolution de la dénomination de l'alliance transfrontalière témoigne du glissement sémantique vers une présentation plus ethnique du projet. L'absence du qualificatif aymara est notamment remarquable dans ses premiers documents de présentation : le nom du regroupement est initialement l'Alliance stratégique trinationale (Alianza estratégica trinacional, AET) des maires ruraux andins de Bolivie, du Chili et du Pérou, Aymaras sans Frontières étant seulement le titre d'un accord signé en 2001 (AET, 2001). Le terme trinational est ensuite éliminé, pour donner officiellement naissance à l'Alliance stratégique Aymaras sans frontières en $2003^{9}$. Suite à la présentation du projet à la BID en 2006, la mise en réseau de l'ASASF est de nature virtuelle et communicationnelle, et fait apparaître le « Monde aymara ». Une revue bimestrielle d'information en ligne est créée en juin 2010, dont le titre, Mundo Aymara, n'est pas sans rappeler l'expérience centraméricaine Mundo Maya, un mégaprojet touristique impulsé par les gouvernements centraméricains en 1992. Si les genèses de ces deux «mondes» diffèrent fortement, l'invocation de ce modèle par le président de l'ASASF lors de notre entretien de 2006 témoigne de sa volonté de ralliement à une cause indigène dont l'écho transnational conditionne en partie le succès politique.

La négation discursive de la frontière s'accompagne de la réactivation d'une mémoire aymara sur l'espace de l'ancien Collasuyo, l'une des quatre parties de l'Empire inca. L'opposition et le jeu entre les deux symboles identitaires que sont le territoire national et le territoire aymara permet d'objectiver l'espace couvert par l’Alliance et de légitimer l'action publique transfrontalière.

9. ASASF, 2003. Cette date coïncide d'ailleurs avec la création légale de l'Alliance stratégique Aymaras sans frontières, sous le statut de corporation privée à but non lucratif. 
Toutefois, une lecture des documents de travail de l'ASASF depuis 1999 permet de constater que l'évocation d'un territoire ne gagne en sens et en légitimité - notamment auprès des gouvernements nationaux - qu'une fois affirmée la dimension culturelle du projet. La mise en scène d'un «espace régional frontalier, avec des potentialités et des limites communes» (AET, 2002a) en tant que territoire transfrontalier débute avec les premières rencontres entre maires ruraux et Banque mondiale (ASASF, 2003), pour finalement donner lieu à une carte du territoire aymara (cf. carte) et à des données statistiques afférentes (ASASF, 2006). Lorsque le peuple aymara fait enfin son apparition dans les documents officiels, il est surtout défini selon des considérations historiques: «Le peuple aymara est défini comme le peuple constitué par des aymaraphones composés historiquement par des sous-groupes, tels que les Qullas, Lupacas, Carangas, Lipez, Collaguas, Soras, Pacajes, entre autres, qui étaient établis quasiment dans toutes les Andes, du sud du Lac Titicaca au nord du Chili et de l'Argentine» (ibid.). Une cosmovision originale (Berg, Schiffers, 1992) distinguerait également la population aymara :

Les principales caractéristiques sociales et humaines de l'homme aymara sont une correspondance avec son environnement rural andin ou Puna américaine et son système de vie est basé sur les principes de rationalité, principes éthiques, complémentarité, réciprocité, temps cyclique, correspondance (univers-homme) et respect de la nature, ce qui constitue la «Cosmovision andine» et l'essence de sa culture. (lbid.)

Le document affirme ensuite la persistance du rôle des autorités originaires ou ancestrales en tant que soutien de ces traditions culturelles, sans différencier les conditions existantes dans les trois pays. Le capital social aymara est ainsi valorisé par l'affirmation de spécificités culturelles, spirituelles et sociopolitiques réinventées sur cet espace transfrontalier, en écho aux désignations transnationales dont les communautés andines font l'objet; sur cette base, ce sont ensuite les dimensions économiques de la culture qui sont réinterprétées afin d'assurer une perspective de développement dans la région.

\section{Le devenir de l'Un. Une marque Aymara pour sécuriser l'altiplano}

Outre l'organisation socio-politique, les dynamiques socio-économiques andines sont investies de discours homogénéisants. Les groupes autochtones andins sont généralement assimilés à des populations pauvres et vulnérables dont le milieu de vie est rural et paysan. Cette perception présente les populations andines comme "se situant en-dehors du cours de l'histoire moderne" (Starn, 1991, p. 64), vivant d'agriculture de subsistance (Shelton, Katrinka éd., 
1995) dans des espaces ruraux dont l'économie ne serait «pas hautement monétarisée» (Vives éd., 2006, p.91). Cette description ignore l'existence, dans le cas aymara, d'une bourgeoisie très active économiquement à la Paz et dans les villes du nord chilien, dont le dynamisme s'appuie sur l'effet notable de la frontière dans cette région et sur des réseaux ville-campagne élaborés. Lorsque ces réseaux sont pris en compte par les bailleurs de fonds, ils sont considérés comme des opportunités pour

capitaliser les avantages comparatifs des ressources naturelles, culturelles et sociales originales des communautés et soutenir les liens progressifs entre les pratiques traditionnelles et modernes. Toutefois, capitaliser ces opportunités nécessite de nouveaux modèles institutionnels, des partenariats stratégiques, des approches innovantes et des formations. (Ibid., p. 95)

Un objectif des programmes de développement destinés aux populations indigènes consiste donc à former tant les populations que les représentants locaux afin que la capitalisation des avantages comparatifs liés à certains traits de la culture andine devienne effective. Pour ce faire, l'invocation du capital social des communautés andines constitue à nouveau un argument central, se déclinant notamment sous la notion de «savoir indigène » considéré comme «partie intégrante de l'écosystème local » et comme le principal atout à valoriser (World Bank, 1998, p. 4).

Ainsi, les pratiques et savoirs locaux sont retenus comme fondements du développement économique. En 2011, le rapport de l'ASASF sur le thème de l'économie et du commerce traditionnel se concentre sur la gestion communautaire et rotative des terres et sur la cosmovision, présentées comme des pratiques à mobiliser en tant que capital social :

Malgré le capital social et culturel que présentent les populations originaires, elles se trouvent parmi la population la plus pauvre de la région. À partir de la proposition de l'ASASF d'orienter des actions qui promeuvent le développement régional à partir de la mise en valeur de ce capital, nous entrevoyons des opportunités de mise à profit durable des ressources dans le contexte du système traditionnel de gestion de celles-ci. (ASASF, 2011, p. 27)

Tant les productions agricoles locales qu'une certaine image de la communauté sont alors étudiées et réinterprétées pour être insérées dans un processus de marchandisation des produits de la culture, qui à son tour est supposée engendrer une (re)production de la culture aymara. La culture transfrontalière est notamment rapportée à deux types de produits à valoriser sur les marchés nationaux et internationaux, devant donner naissance au label «Aymara »: ceux qui sont issus de la production locale, et les produits culturels et naturels destinés aux marchés touristiques, comme le montre le tableau ci-dessous : 
Tableau 1. Programme «économie et commerce traditionnel» du Plan stratégique de l'ASAF, 2011

\begin{tabular}{l|l|c}
\hline Numéro & Nom, profil de projet (format BID) & Montant (US \$) \\
\hline 1 & $\begin{array}{l}\text { Renforcement de l'identité aymara et de la gestion } \\
\text { territoriale dans le cadre de la reconstruction de relations } \\
\text { de complémentarité dans la zone rurale d'Amayras sans } \\
\text { frontières }\end{array}$ & 190800 \\
\hline 2 & $\begin{array}{l}\text { Identification de pratiques culturelles positives et } \\
\text { génération de capacités pour la gestion de projets de } \\
\text { tourisme communautaire dans le contexte du Monde aymara }\end{array}$ & 68000 \\
\hline 3 & $\begin{array}{l}\text { Éléments pour la construction d'une stratégie de valorisation } \\
\text { de la culture aymara dans un contexte d'interculturalité }\end{array}$ & 16500 \\
\hline 4 & $\begin{array}{l}\text { Mise en valeur des ferias traditionnelles comme complément } \\
\text { à l'offre touristique du Monde aymara }\end{array}$ & 334000 \\
\hline & Sous-total & 609300 \\
\hline
\end{tabular}

Source : ASASF, 2011, p. 41.

Le projet élaboré pour la BID a donc contribué à élargir les aires d'action de l'ASASF, depuis une cible touristique jusqu'à la création d'un programme englobant la région et ses activités dans leur ensemble, sous le vocable de patrimoine naturel et culturel. Le développement touristique reste tout de même prédominant dans le budget prévisionnel global, le Plan soulignant l'inexistence, dans l'ensemble de la zone, d'une véritable offre touristique qui mettrait en valeur la culture aymara. Le lien établi entre patrimoine, tourisme et culture a pour implication une négation, en discours, du rapport marchand en même temps qu'une valorisation symbolique du produit. Cette partie du Plan est aussi celle qui valorise de la manière la plus évidente la notion de culture aymara :

Nous prétendons que l'activité touristique est capable de générer des effets positifs sur la culture, en conservant de manière appropriée l'environnement et la société, ce qui contribue au bien-être de la société de manière complémentaire à d'autres activités économiques comme l'agriculture, l'élevage, l'artisanat, le commerce, les services, entre autres, sur un territoire géographique qui présente une grande diversité d'écosystèmes et une précieuse richesse culturelle. (Ibid., p. 22)

Les savoirs traditionnels, la cosmovision andine et les rapports rituels et ancestraux à la nature et à la terre-mère (Pachamama) constitueraient une forme efficace de gestion des ressources naturelles et une clé du développement durable et de la conservation de la biodiversité (Davis, Ebbe, 1995). Ce lien préétabli entre conservation de la culture indigène et préservation de la nature se trouve ici au cœur de la notion de patrimoine. L'activité touristique, par l'effet d'une «mise en suspens du monde ordinaire» et la création d'un « rapport enchanté au monde » (Réau, Poupeau, 2007) devrait donc in fine contribuer à une conservation de l'environnement par la valorisation des activités traditionnelles. 
Le patrimoine aymara est aussi et plus fondamentalement transformé en Bien public régional. Les Biens publics régionaux sont des programmes considérés par les institutions internationales comme des leviers pour le transfert de bonnes pratiques entre États d'une même région, et qui doivent se substituer à la conditionnalité des prêts directs aux États. Dans le cas de l'ASASF, l'État central chilien a été le principal moteur de légitimation du projet, notamment dans l'objectif d'une homologation des normes de sécurité frontalière entre les trois États. Ces dynamiques ayant été analysées en détail par ailleurs (Rouvière, 2012, 2014), soulignons ici la manière dont la thématique de la sécurité est intégrée aux discours des maires chiliens, ce relais local des normes de sécurité constituant un élément indispensable à la mise en ordre de l'espace transfrontalier via les politiques de développement. Au-delà du rapprochement entre tourisme, culture et nature, la perspective du développement avec identité permet effectivement de générer une visibilité sur les activités transfrontalières. L'un des effets attendus des liens établis entre la construction du territoire aymara et l'accès à des niches de marché pour les populations locales est en effet d'insérer ces dernières dans les marchés formels. Le maire de Pica souligne ainsi que le projet ASASF permettra de modifier ces comportements en substituant aux flux illégaux transfrontaliers la création d'entreprises dans la zone :

Je crois que ce projet est très bon, parce qu'il va permettre à ces communautés, qui sont si petites, de pouvoir commercialiser leurs produits [...], d'avoir leurs papiers à jour, de pouvoir produire des factures, de pouvoir vendre leurs produits, et aussi de vendre leurs produits comme il se doit. [...] et vraiment, chaque pays va devoir se préoccuper de vendre ses produits à l'étranger, mais comme il se doit. ${ }^{10}$

Ce type de production de la culture doit donc avoir pour effet de dévier les populations frontalières des marchés informels entre les trois pays, en générant des opportunités légales.

Mais plus encore que l'informalité ou la contrebande, la suspicion permanente envers les Aymaras pour leur participation à des activités telles que le trafic de drogue et les difficultés concrètes des États à établir des contrôles dans la région génèrent un type de contrôle plus indicible, celui d'un développement destiné à lutter contre la tentation du terrorisme. L'association entre menace terroriste et pratiques transfrontalières indigènes repose sur une analyse approximative des statistiques policières et une vision souvent fantasmée de l'insurrection indienne. Si les populations frontalières pratiquent diverses activités économiques informelles et sont parfois recrutées pour jouer le rôle de mules afin de transporter la drogue entre la Bolivie et le Chili, elles ne sont pas les principaux acteurs du trafic transfrontalier ${ }^{11}$. Le lien établi avec des pra-

10. Entretien avec le maire de Pica (Chili), 2006.

11. Gendarmería de Chile; Corder, 2009. 
tiques terroristes est la manifestation d'amalgames évidents, qui orientent pourtant certaines actions menées sur la triple frontière andine. Ce discours surl'idée d'une hypothétique menace terroriste est relayé pour la première fois en 2002 lors d'un événement tenu à Tacna (Pérou) et financé par la Banque mondiale (AET, 2002b). Lors de divers entretiens avec la presse locale, le président de l'ASASF brandit ce spectre pour justifier les plans de développement transfrontaliers. Il évoque comme préoccupation principale de l'Alliance «la recrudescence du terrorisme en Amérique latine »; après avoir souligné la persistance du Sentier lumineux au Pérou, il continue ainsi : "Cela arrive dans le sud du Chili, dans le monde mapuche. En Colombie nous avons un problème similaire. [...] La manière de contrer cela est de créer un pôle de développement dans toute la macro-région du monde aymara. » ${ }^{12}$ L'élaboration de politiques de développement avec identité contribue paradoxalement à soutenir un discours sécuritaire, sécurité et développement devant se renforcer mutuellement. Le point nodal de ce lien réside dans les perspectives renouvelées d'identification au groupe aymara et des bénéfices économiques qui y sont potentiellement liés. L'identité, telle qu'elle est construite dans les discours partagés des acteurs locaux et transnationaux sur le développement, devient un gage de sécurisation dans cette région andine, plus encore qu'une garantie d'amélioration des conditions socio-économiques locales.

Les dirigeants des municipalités andines et frontalières présentent de réelles capacités d'adaptation stratégique aux réquisits des bailleurs de fonds internationaux. La centralité du référentiel discursif de l'ethnicité renvoie à ce que J.-P. Lavaud (2005) a nommé les indianismes occidentaux, soit des particularismes politiques dont le contenu, voire l'existence, sont fortement déterminés par la présence de sources de coopération externes. Mais l'aymarité transfrontalière et transnationale se présente également comme un levier discursif pour une mise en ordre politique et économique de cet espace diplomatiquement contesté. Les revendications locales et indigènes se retrouvent ainsi réinsérées dans des logiques gouvernementales plus larges. En l'occurrence, le projet de l'ASASF répond aux exigences de l'intégration régionale et de la sécurisation des flux de marchandise sur le continent, l'un des couloirs bi-océaniques devant notamment traverser cet espace. Si le futur de cette Alliance est incertain - les activités étant largement réduites depuis la fin de l'intervention de la BID en 2011 -, le processus transfrontalier sur une décennie a toutefois débouché sur un enrôlement des dirigeants locaux dans un discours permettant la sécurisation de ces zones rurales, via la reconnaissance de spécificités culturelles considérées comme les bases d’un futur bien-être socio-économique des populations autochtones.

12. «Amenaza terrorista avanza en zonas rurales de América », El Correo, Tacna, 24 mars 2002. 
Le soutien technique et financier des bailleurs de fonds aux projets locaux constitue une forme de reconnaissance, les autorités autochtones devant savoir jouer d'enjeux dépassant les problématiques locales pour légitimer leur action. Ici, l'une des conditions qui mènent à l'insertion du projet dans des considérations de sécurisation de l'espace considéré réside dans la mise en marche et en marché de l'identité aymara. La revendication d'identités autochtones en Amérique latine ne renvoie donc pas nécessairement aux mobilisations progressistes les plus visibles (Poupeau, Do Alto, 2009; Gros, 2003). Elle peut répondre à des logiques stratégiques, de nature politique et économique, mais contient également un potentiel de mobilisation et d'usages discursifs renouvelés qui modifient, à leur tour, les cadres dont a émergé l’identité. Dans le cas aymara et transfrontalier, les usages de ces cadres esquissés restent à observer.

\section{Références}

\section{Bibliographie}

Albó Xavier, 2000, «Aymaras entre Bolivia, Perú y Chile », Estudios atacameños, nº19, p. 43-73.

AlvizURI Verushka, 2012, Le savant, le militant et l'Aymara. Histoire d'une construction identitaire en Bolivie (1952-2006), Paris, Armand Colin.

Andolina Robert, LaURie Nina, Radcliffe Sarah A., 2009, Indigenous Development in the Andes. Culture, Power and Transnationalism, Durham, Londres, Duke University Press.

BARTH Fredrik, 1995, Les groupes ethniques et leurs frontières, Paris, PUF.

BALANDIER Georges, 1980, Pouvoir sur scène, Paris, PUF.

BELLIER Irène, 2012, «Les peuples autochtones aux Nations Unies. La construction d'un sujet de droit / acteur collectif et la fabrique de normes internationales», Critique internationale, $\mathrm{n}^{\circ} 54, \mathrm{p} .61-80$.

Berg Hans, SchIffERs Norbert éd., 1992, La cosmovisión aymara, La Paz, HISBOL/UCB, p. 143-186.

BOURDIEU Pierre, 1980, "L'identité et la représentation, éléments pour une réflexion critique sur l'idée de région », Actes de la recherche en sciences sociales, n³5, p. 63-72.

BouysSe-CASSAgne Thérèse, 1987, La identidad aymara. Aproximación histórica (siglo xv, siglo XVI), La Paz, HISBOL/IFEA.

BRES Jacques, NowAKowSKA Aleksandra, 2005, «Dis-moi avec qui tu “dialogues”, je te dirai qui tu es... De la pertinence de la notion de dialogisme pour l'analyse du discours», Marges linguistiques, ${ }^{\circ}$ 9, mai, p. 137-153.

Charaudeau Patrick, 2009, "Identité sociale et identité discursive. Un jeu de miroir fondateur de l'activité langagière», Identités sociales et discursives du sujet parlant, P. Charaudeau éd., Paris, L'Harmattan. 
Do Alto Hervé, Stefanon Pablo, 2008, Nous serons des millions. Evo Morales et la gauche au pouvoir en Bolivie, Paris, Raisons d'agir.

GALINIER Jacques, Mo LINIÉ Antoinette, 2006, Les Néo-Indiens. Une religion du III millénaire, Paris, Odile Jacob.

GoffMAn Erving, 1975, Stigmate. Les usages sociaux des handicaps, Paris, Minuit.

Gros Christian, 2003, "Demandes ethniques et politiques publiques en Amérique latine», Problèmes d'Amérique latine, ${ }^{\circ}$ 48, printemps, p. 11-29.

HÉRITIER Françoise, 1977, "L’identité Samo », L'identité. Séminaire interdisciplinaire dirigé par Claude Lévi-Strauss, professeur au Collège de France, 1974-1975, C. LeviStrauss éd., Paris, Grasset, p. 51-71.

HıBou Béatrice, 1998, "Économie politique du discours de la Banque mondiale en Afrique subsaharienne. Du catéchisme économique au fait (et méfait) missionnaire », Les Études du CERI, $\mathrm{n}^{\circ} 39$, mars.

НовSваWм Éric, 1995, «Inventing traditions », Enquête, n² 2, p. 171-189.

LAVAUd Jean-Pierre, 2005, "Démocratie et ethnicisation en Bolivie », Problèmes d'Amérique latine, $\mathrm{n}^{\circ} 56$, printemps, p. 105-128

LAVAUd Jean-Pierre, LeSTAGE Françoise, 2006, «L'indianisme en Amérique latine. Historique, réseaux, discours, effets pervers », Esprit, janvier, p. 42-64.

Maingueneau Dominique, 2010, "Le discours politique et son "environnement" ", Mots. Les langages du politique, n²9, Trente ans d'étude des langages du politique (1980-2010), p. 85-90.

MORIN Françoise, SANTANA Roberto, 2003, Lo transnacional. Instrumento y desafío para los pueblos indígenas, Quito, Abya-Yala.

Poupeau Frank, Do Alto Hervé, 2009, "L'indianisme est-il de gauche? Remarques complémentaires sur l'Occident décroché », Civilisations, nº58 (1), p.141-147.

RAdCliffe Sarah, LAURIE Nina, 2006, "Culture and development. Taking culture seriously in development for Andean indigenous people», Environment and Planning D. Society and Space, $\mathrm{n}^{\circ} 24$, p. 231-248.

RÉAu Bertrand, PoupeAu Franck, 2007, "L'enchantement du monde touristique », Actes de la recherche en sciences sociales, vol. V, nº170, p. 4-13.

RouvIÈRE Lætitia, 2012, À la frontière de l'État. Gouvernement et territorialités aymaras au Chili, thèse de doctorat, Institut d'études politiques, Université de Grenoble.

- 2014, "Gobernar territorialidades transfronterizas. Seguridad y "desarrollo con identidad” aymara en la triple frontera del norte de Chile (Chile-Perú-Bolivia)», Trace, ${ }^{\circ} 65$, juin, p. 37-50.

Rouvière Lætitia, Perrier-Bruslé Lætitia, 2015, «Chilean northern borders with Peru and Bolivia. Territorial dispute and structural interdependence on the Pacific Coast », Border Disputes. A Global Encyclopedia, E. Brunet Jailly éd., Santa Barbara, ABC Clio.

STARN Orin, 1991, «Missing the revolution. Anthropologists and the war in Peru », Cultural Anthropology, $n^{\circ} 6$ (1), février, p. 63-91.

StIGLITZ Joseph, 1998, «More instruments and broader goals. Moving towards the Post-Washington Consensus », Wider Annual Lectures, Helsinki, World Institute for Development Economics Research. 


\section{Documents institutionnels}

AliAnZA eStratÉGICA TRINACIONAL, 2001, «Aymaras... Sin fronteras. Alianza estratégica Bolivia-Chile-Perú por nuestras fronteras andinas ", 9-10 novembre, Putre.

- 2002a, «Estructura y funciones de la Alianza Estratégica Trinacional», Tacna, 22 mars.

- 2002b, «Acta de Reunión. I Convención Alianza Estratégica Trinacional (AET) de los presidentes de las asociaciones municipales rurales andinas de Bolivia, Chile, Perú. 23 Marzo de $2002 »$.

ASASF, 2003, «Modelo de desarrollo de integración para los gobiernos locales transfronterizos» (Document interne).

- 2006, «Alianza estratégica : modelo de integración transfronterizo en Sudamérica », Informe Gestión 2001-2006.

— 2007, «Alianza estratégica “Aymaras sin fronteras”. Modelo de integración transfronterizo en Sudamerica », Document interne, septembre.

- 2011, «Plan estratégico Aymaras sin fronteras. Programa "Recuperación, fomento y puesta en valor del patrimonio cultural y natural Aymara”, ATN/OC-107-RG ».

BANCO INTERAMERICANO DE DESARRollo, 2007, «Recuperación, fomento y puesta en valor del patrimonio cultural y natural Aymara (RG-T1271). Plan de operaciones» (Document interne).

BANCO MUNDIAL, 1987, Informe sobre el desarrollo mundial.

- 1991, La tarea acuciante del desarrollo. Resumen del Informe sobre el desarrollo mundial 1991, Washington.

- 2002, "La estrategia sobre municipalidades indígenas. Una propuesta de empoderamiento para los Aymara ", Informe sobre la Misión de Lanzamiento del Proyecto para el Fortalecimiento de la Red de Municipalidades Aymaras, Arica-Chile, 29-31 janvier 2002 (Document interne).

CORDER Alejandro, 2009, «Infracciones penales en espacios de frontera. El narcotráfico en la provincia del Tamarugal años 2000-2009», Proyecto de investigación interno (UNAP) DI 0064-09.

Davis Shelton, EbBe Katrinka éd., 1995, Traditional Knowledge and Sustainable Development, Proceedings of a Conference held at the World Bank, Washington DC, 27-28 septembre 1993.

Deruyttere Anne éd., 2006, Política operativa sobre pueblos indígenas y Estrategia para el desarrollo indígena, Washington, Banco Interamericano de Desarrollo, p. 94 .

PARTRIDGE William L., UquILLAS Jorge E., Johns Kathryn, 1996, Including the Excluded: Ethnodevelopment in Latin America, World Bank.

VIVES Antonio éd., 2006, Sustaining development for all. Expanding Access to Economic Activity and Social Services, BID.

WORLD BANK, 1998, Indigenous Knowledge for Development. A Framework for Action, Knowledge and Learning Center, Africa Region, World Bank. 